\title{
Immuno-inflammatory Characters in Low Severity COVID-19 Patients with Digestive Symptoms
}

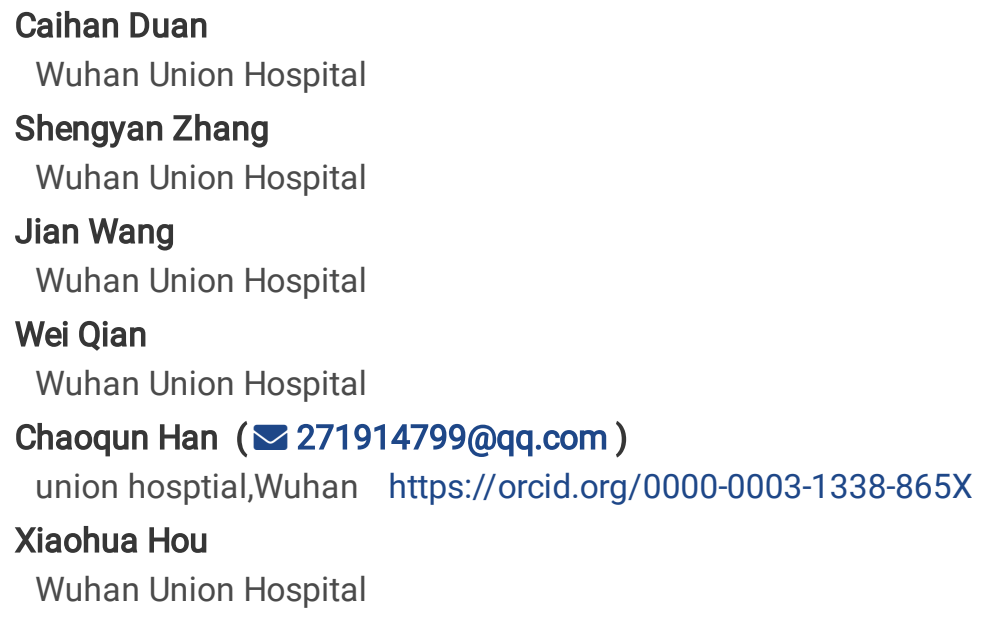

Research article

Keywords: COVID-19; digestive symptoms; mild; inflammatory immune system

Posted Date: May 12th, 2020

DOI: https://doi.org/10.21203/rs.3.rs-23267/v1

License: (c) (i) This work is licensed under a Creative Commons Attribution 4.0 International License. Read Full License 


\section{Abstract}

Background: Coronavirus disease 2019 (COVID-19) is an emerged infection raised widely concerns for pneumonia and respiratory manifestations. It is noteworthy that digestive symptoms are frequently observed in COVID-19 patients. We sought to describe the immune-inflammatory characteristics of COVID-19 patients with digestive symptoms and mild disease severity.

Methods: We designated enrolled mild patients into three subtypes depending on the patients with or without digestive symptoms, including Digestive only (digestive symptoms only), Respiratory only (respiratory symptoms only) and Digestive+ Respiratory (Both digestive and respiratory symptoms). Patient discharge was based on negative results of rRT-PCR testing for SARS-CoV-2 from at least two sequential respiratory tract specimens collected $\geq 24$ hours apart. The multiorgan function, immune-inflammatory characteristics were analyzed among three groups.

Results: Mild liver damage and the activation of immuno-inflammatory system are the most abnormalities in mild patients but no significant differences were found ( $\mathrm{p} \otimes 0.05$ ). Compared with the Respiratory only group, patients with digestive symptoms were more likely to have slightly higher and later peak values of inflammatory cytokines during the subsequent course of disease $(P \otimes$ 0.05). Additionally, we also found that there was a significant correlation between IL-2 and TNF level in the Digestive only cases (P区 0.05).

Conclusions: Mild patients only/accompanied with digestive symptoms are a special subtype of COVID-19. Patients in this group were more likely to have slightly higher and later peak values of inflammatory cytokines during the subsequent course of disease. The prevention and clinical management of this type should be taken into consideration.

\section{Introduction}

The Coronavirus disease 2019 (COVID-19) has spread throughout worldwide now and has been declared an international public health emergency by the World Health Organization (WHO)[1, 2]. Respiratory symptoms including cough, shortness of breath, dyspnea etc. are considered as common clinical manifestations of COVID-19 [3]. However, Series of recent researches have reported digestive symptoms, such as nausea, vomit and diarrhea were also as accompanied or only symptoms of the patients [4, 5].

The virus target cell receptor, angiotensin converting enzyme 2 (ACE2), was highly expressed in gastrointestinal organs such as small intestine and duodenum, providing the foundation of gastrointestinal infection[6]. On the other hand, the viral nucleic acid could also be detected in the feces. $23.29 \%$ of patients had positive stools after negative respiratory samples, indicating "cured" patients may still result in the damage[7, 8]. The digestive involvement of 2019-nCoV should be recognized.

Actually, although the virus is sweeping the worldwide, $80.9-86.0 \%$ of patients were with mild severity[9]. More importantly, mild patients facilitate the rapid dissemination of 2019-nCoV and are the main cause of the spread infection[10]. Therefore, understanding the characters of this group could help for treating and controlling the dissemination at early stage. Our previous study has shown that patients with digestive symptoms are more likely to have a longer delay before viral clearance compared to patients with only respiratory symptoms[11, 12]. However, the underlying reasons remain largely unknown.

In highly pathogenic human coronaviruses (hCoVs) such as severe acute respiratory syndrome CoV (SARS-CoV) and middle east respiratory syndrome CoV (MERS-CoV) infection, cytokine storm and immunosuppression led to deteriorate clinical results[13]. Similarly, it is confirmed that severe COVID-19 cases had markedly higher levels of inflammatory cytokines and lower absolute number of T lymphocytes[14]. Further research showed SARS-CoV-2 virus infection led to lymphopenia and cytokine storm which correlated with disease severity[15]. Nevertheless, the inflammatory and immune characters in patients with digestive symptoms and mild severity is largely unknown. On this account, the present study is aim to illustrate the immuno-inflammatory characteristics and their longitudinally variations in mild patients.

\section{Methods}

\section{Patient diagnosis and Groups}


The study was done at Union Hospital, Tongji Medical College (Wuhan, China), which was a designated hospital to management of patients with COVID-19. We enrolled 206 mild patients during February 13th to February 29th, 2020, through laboratoryconfirmed by real-time reverse-transcriptase polymerase-chain-reaction (RT-PCR) assay for nasal and pharyngeal swab specimens[16], which was conducted in accordance with the protocol established by the World Health Organization[17]. Mild patients were defined as patients without dyspnea, without clinical evidence of respiratory distress, and able to maintain blood oxygen saturation above $93 \%$ in resting condition[3, 11]. Based on whether having digestive (nausea, vomit, diarrhea and anorexia,) and/or respiratory symptoms (cough/expectoration, chest tightness, pharyngalgia, shortness of breath), patients were divided into three groups, named "Digestive only," "Respiratory only" and "Digestive + Respiratory".

This study was approved by the Medical Ethical Review Committee, Union Hospital of Tongji Medical College, Huazhong University of Science and Technology, China ([2020] No.0033). Written informed consent was waived due to the rapid emergence of this infectious disease and their information had been anonymized and de-identified.

\section{Data Collection}

In order to obtain more accurate gastrointestinal symptoms of patients, we conducted a telephone survey and face to face to obtain more detailed information. We also interviewed patients who had only respiratory symptoms to make sure they did not have gastrointestinal symptoms. The epidemiological, clinical manifestation, laboratory, and outcome data was extracted from medical and nursing records. Laboratory assessments consisted of complete blood count, stool routine, blood chemistry (including electrolytes, liver and renal function, lactate dehydrogenase and creatine kinase, etc.), coagulation test, inflammatory biomarkers (including C-reactive protein, erythrocyte sedimentation rate (ESR), procalcitonin, IL-2, IL-4, IL-6, IL-10, TNF, etc.), immunity index (including percentage of NK cells, B /CD3 ${ }^{+} / \mathrm{CD} 4^{+} / \mathrm{CD} 8^{+}$lymphocytes, C3, C4, IGE, IGG, IGM, etc.). All data were calculated from the onset date of disease and checked by two trained doctors. The clinical data were monitored up to March 18, 2020, the final date of follow-up.

\section{Statistical analysis}

Categorical variable results are described as numbers and percentages. Continuous variables are described as mean \pm standard deviation (SD), means, maximums and minimums as appropriate. Chi-square tests and Fisher's exact tests were used for categorical variables. Wilcoxon rank-sum tests and Pearson correlation analysis were applied to continuous variables as appropriate. For the results with suggested correlation, a scatter graph was further drawn. Then linear regression analysis was carried out to calculate $\mathrm{R}^{2}$ and $\mathrm{P}$ values. Statistical analysis was performed using IBM SPSS Statistics software (version 20.0, IBM Corp, Armonk, NY, USA). P $\leq 0.05$ was considered statistically significant (two-sided).

\section{Results}

\section{General clinical characteristics}

In the 206 full cohort, all patients were residents of Wuhan with a median age of 62.5 years (ranged from 27 to 92 years). 89 $(43.2 \%)$ were younger than 60 years and most patients are retirees (68.9\%) and female (55.8\%). Hypertension (27.2\%), diabetes $(10.2 \%)$ and cardiovascular disease $(12.7 \%)$ were the most common comorbidity in COVID-19 patients. There were 48 patients of Digestive only (23.3\%), 89 patients of Respiratory only (43.2\%) and 69 cases of Digestive + Respiratory (33.5\%), respectively. A series of respiratory symptoms were including cough/expectoration (25.7\%), chest tightness (23.8\%), shortness of breath (14.6\%), and pharyngodynia (6.4\%). Of digestive symptom patients, 67 patients $(57.3 \%)$ were presented with diarrhea. Patients who reported diarrhea were more likely to have a fever in Digestive only group $(13 / 23,56.5 \%$ vs. $6 / 25,24.0 \%, P=0.021)$; and were younger (49.5y vs. 61.4y, $\mathrm{P}=0.001$ ) in Digestive + Respiratory group (Table 1). 
Table 1

Clinical characteristics based on the classification of digestive symptoms with or without diarrhea of Digestive only and Digestive + Respiratory patients on admission.

\begin{tabular}{|c|c|c|c|c|c|c|c|c|c|}
\hline \multirow[t]{2}{*}{ Items } & \multicolumn{3}{|c|}{$\begin{array}{l}\text { Total(including } \\
\text { Respiratory only) }\end{array}$} & \multicolumn{3}{|c|}{ Digestive only } & \multicolumn{3}{|c|}{ Digestive + Respiratory } \\
\hline & $\begin{array}{l}\text { With } \\
\text { Diarrhea } \\
(\mathrm{N}=67)\end{array}$ & $\begin{array}{l}\text { Without } \\
\text { Diarrhea } \\
(\mathrm{N}=139)\end{array}$ & $\mathbf{P}$ & $\begin{array}{l}\text { With } \\
\text { Diarrhea } \\
(\mathrm{N}=23)\end{array}$ & $\begin{array}{l}\text { Without } \\
\text { Diarrhea } \\
(\mathrm{N}=25)\end{array}$ & $\mathbf{P}$ & $\begin{array}{l}\text { With } \\
\text { Diarrhea } \\
(\mathrm{N}=44)\end{array}$ & $\begin{array}{l}\text { Without } \\
\text { Diarrhea } \\
(\mathrm{N}=25)\end{array}$ & $\mathbf{P}$ \\
\hline Age (years) & $\begin{array}{l}51.6(27- \\
84)\end{array}$ & $\begin{array}{l}62.0(27- \\
92)\end{array}$ & 0.128 & $\begin{array}{l}55.5(32- \\
84)\end{array}$ & $\begin{array}{l}67.6(32- \\
92)\end{array}$ & 0.424 & $\begin{array}{l}49.5(27- \\
81)\end{array}$ & $\begin{array}{l}61.4(36- \\
84)\end{array}$ & 0.001 \\
\hline $\begin{array}{l}\text { Gender } \\
\text { (Male/Female) }\end{array}$ & $23 / 44$ & $68 / 71$ & 0.048 & $6 / 17$ & $7 / 18$ & 0.882 & $17 / 27$ & $13 / 12$ & 0.282 \\
\hline Fever & $48(71.6 \%)$ & $90(64.7 \%)$ & 0.324 & $13(56.5 \%)$ & $6(24.0 \%)$ & 0.021 & $35(79.5 \%)$ & 19(76.0\%) & 0.731 \\
\hline $\begin{array}{l}\text { Highest } \\
\text { temperature, }{ }^{\circ} \mathrm{C}\end{array}$ & 39.8 & 39.8 & - & 39 & 39.8 & - & 39.8 & 39.5 & - \\
\hline \multicolumn{10}{|l|}{$\begin{array}{l}\text { Organ function } \\
\text { injury }\end{array}$} \\
\hline $\begin{array}{l}\text { TBil }(\leq \\
19.0 \mu \mathrm{mol} / \mathrm{L})\end{array}$ & $7(10.4 \%)$ & $10(7.2 \%)$ & 0.436 & $2(8.7 \%)$ & $2(8.0 \%)$ & 1 & $5(11.4 \%)$ & $2(8.0 \%)$ & 1 \\
\hline $\begin{array}{l}\operatorname{DBil}(\leq \\
6.8 \mu \mathrm{mol} / \mathrm{L})\end{array}$ & $6(9.1 \%)$ & $15(10.9 \%)$ & 0.696 & $3(13.0 \%)$ & $0(0.0 \%)$ & 0.205 & $3(7.0 \%)$ & $2(8.0 \%)$ & 1 \\
\hline $\operatorname{ALT}(\leq 35 \mathrm{U} / \mathrm{L})$ & $17(25.4 \%)$ & $54(39.1 \%)$ & 0.052 & $7(30.4 \%)$ & $8(32.0 \%)$ & 0.907 & $10(22.7 \%)$ & $9(36.0 \%)$ & 0.235 \\
\hline $\mathrm{AST}(\leq 40 \mathrm{U} / \mathrm{L})$ & $6(9.0 \%)$ & $25(18.1 \%)$ & 0.086 & $2(8.7 \%)$ & $5(20.0 \%)$ & 0.484 & $4(9.1 \%)$ & $6(24.0 \%)$ & 0.182 \\
\hline $\operatorname{ALP}(<150 U / L)$ & $0(0.0 \%)$ & $5(3.6 \%)$ & 0.274 & $0(0.0 \%)$ & $2(8.0 \%)$ & 0.508 & $0(0.0 \%)$ & $0(0.0 \%)$ & - \\
\hline GGT $(<50 \mathrm{U} / \mathrm{L})$ & $16(23.9 \%)$ & $29(21.0 \%)$ & 0.642 & $4(17.4 \%)$ & $6(24.0 \%)$ & 0.836 & $12(27.3 \%)$ & $3(12.0 \%)$ & 0.139 \\
\hline $\mathrm{LDH}(<245 \mathrm{U} / \mathrm{L})$ & $23(34.3 \%)$ & $46(34.1 \%)$ & 0.971 & $9(39.1 \%)$ & $10(40.0 \%)$ & 0.951 & 14(31.8\%) & $8(32.0 \%)$ & 0.988 \\
\hline $\begin{array}{l}\text { hsTNI (< } \\
26.2 \mathrm{ng} / \mathrm{L})\end{array}$ & $0(0.0 \%)$ & $4(3.5 \%)$ & 0.463 & $0(0.0 \%)$ & $1(4.8 \%)$ & 1 & $0(0.0 \%)$ & $0(0.0 \%)$ & - \\
\hline $\begin{array}{l}\text { D-Dimer (< } \\
0.5 \mathrm{ng} / \mathrm{L})\end{array}$ & $25(45.5 \%)$ & $57(52.3 \%)$ & 0.408 & $7(36.8 \%)$ & $12(75.0 \%)$ & 0.041 & $18(50.0 \%)$ & $11(50.0 \%)$ & 1 \\
\hline \multicolumn{10}{|l|}{$\begin{array}{l}\text { Immune } \\
\text { inflammation }\end{array}$} \\
\hline $\begin{array}{l}\text { WBC (3.5- } \\
9.5 \mathrm{G} / \mathrm{L})\end{array}$ & $7(10.4 \%)$ & $29(21.2 \%)$ & 0.059 & $2(8.7 \%)$ & $4(16.7 \%)$ & 0.666 & $5(11.4 \%)$ & $6(24.0 \%)$ & 0.3 \\
\hline $\begin{array}{l}\text { Neutrophil (1.8- } \\
6.3 \mathrm{G} / \mathrm{L})\end{array}$ & $7(10.4 \%)$ & $28(20.4 \%)$ & 0.075 & $3(13.0 \%)$ & $5(20.8 \%)$ & 0.701 & $4(9.1 \%)$ & $6(24.0 \%)$ & 0.182 \\
\hline $\begin{array}{l}\text { Lymphocyte(1.1- } \\
\text { 3.2G/L) }\end{array}$ & $18(26.9 \%)$ & $52(38.0 \%)$ & 0.117 & $6(26.1 \%)$ & $10(41.7 \%)$ & 0.260 & $12(27.3 \%)$ & $7(28.0 \%)$ & 0.948 \\
\hline CRP $(\llbracket 8 \mathrm{mg} / \mathrm{L})$ & $21(38.9 \%)$ & $66(54.5 \%)$ & 0.056 & $7(36.8 \%)$ & $9(37.5 \%)$ & 0.965 & $14(40.0 \%)$ & $9(45.0 \%)$ & 0.718 \\
\hline ESR $(\nabla 15 \mathrm{~mm} / \mathrm{h})$ & $40(71.4 \%)$ & 103(84.4\%) & 0.043 & 14(77.8\%) & $20(90.9 \%)$ & 0.381 & $26(68.4 \%)$ & 19(86.4\%) & 0.122 \\
\hline $\begin{array}{l}\mathrm{IL}-2(0.1- \\
4.1 \mathrm{pg} / \mathrm{ml})\end{array}$ & $3(4.8 \%)$ & $1(0.8 \%)$ & 0.214 & $3(13.0 \%)$ & $1(4.2 \%)$ & 0.348 & $0(0.0 \%)$ & $0(0.0 \%)$ & - \\
\hline
\end{tabular}

Indicators of organ function injury and immune inflammation were expressed in terms of the number and percentage of abnormalities; Digestive Only: gastrointestinal symptoms cases; Respiratory Only: respiratory symptoms cases; Digestive + Respiratory: both digestive and respiratory symptoms. Because not all patients have the same test, the denominators of each indicator may be inconsistent in their calculations. A significance level of $P \leq 0.05$ was used. 


\begin{tabular}{|c|c|c|c|c|c|c|c|c|c|}
\hline \multirow{2}{*}{$\begin{array}{l}\text { Items } \\
\begin{array}{l}\text { IL-4(0.1- } \\
3.2 \mathrm{pg} / \mathrm{ml})\end{array}\end{array}$} & \multicolumn{3}{|c|}{$\begin{array}{l}\text { Total(including } \\
\text { Respiratory only) }\end{array}$} & \multicolumn{3}{|c|}{ Digestive only } & \multicolumn{3}{|c|}{ Digestive + Respiratory } \\
\hline & $4(6.3 \%)$ & $11(8.8 \%)$ & 0.558 & $2(8.7 \%)$ & $5(20.8 \%)$ & 0.416 & $2(5.0 \%)$ & $1(5.0 \%)$ & 1 \\
\hline $\begin{array}{l}\text { IL-6(0.1- } \\
2.9 \mathrm{pg} / \mathrm{ml})\end{array}$ & $59(93.7 \%)$ & $119(95.2 \%)$ & 0.918 & $20(87.0 \%)$ & $23(95.8 \%)$ & 0.348 & $39(97.5 \%)$ & $18(90.0 \%)$ & 0.255 \\
\hline $\begin{array}{l}\mathrm{IL}-10(0.1- \\
5.0 \mathrm{pg} / \mathrm{ml})\end{array}$ & $6(9.5 \%)$ & $20(16.0 \%)$ & 0.225 & $3(13.0 \%)$ & 8(33.3\%) & 0.101 & $3(7.5 \%)$ & $2(10.0 \%)$ & 1 \\
\hline $\begin{array}{l}\text { TNF-a (0.1- } \\
23 \mathrm{pg} / \mathrm{ml})\end{array}$ & $0(0.0 \%)$ & $6(4.8 \%)$ & 0.184 & $0(0.0 \%)$ & $0(0.0 \%)$ & - & $0(0.0 \%)$ & $0(0.0 \%)$ & - \\
\hline $\begin{array}{l}\operatorname{lgG}(7.51- \\
15.6 \mathrm{~g} / \mathrm{L})\end{array}$ & $3(13.0 \%)$ & $3(6.0 \%)$ & 0.576 & $2(15.4 \%)$ & $1(6.7 \%)$ & 0.583 & $1(10.0 \%)$ & $1(25.0 \%)$ & 0.505 \\
\hline $\begin{array}{l}\lg M(0.46- \\
3.04 \mathrm{~g} / \mathrm{L})\end{array}$ & $1(4.3 \%)$ & $4(8.0 \%)$ & 0.940 & $0(0.0 \%)$ & $0(0.0 \%)$ & - & $1(10.0 \%)$ & $0(0.0 \%)$ & 1 \\
\hline $\begin{array}{l}\text { C3(0.79- } \\
1.52 \mathrm{~g} / \mathrm{L})\end{array}$ & $3(13.0 \%)$ & $14(28.0 \%)$ & 0.160 & $2(15.4 \%)$ & $4(26.7 \%)$ & 0.655 & $1(10.0 \%)$ & $1(25.0 \%)$ & 0.505 \\
\hline $\begin{array}{l}\mathrm{C} 4(0.16- \\
0.38 \mathrm{~g} / \mathrm{L})\end{array}$ & $3(13.0 \%)$ & $13(26.0 \%)$ & 0.214 & $0(0.0 \%)$ & $4(26.7 \%)$ & 0.102 & $3(30.0 \%)$ & $2(50.0 \%)$ & 0.580 \\
\hline $\begin{array}{l}\text { CD3 + } \\
\text { Tlymphocytes }\end{array}$ & $19(30.6 \%)$ & $30(22.4 \%)$ & 0.214 & $7(31.8 \%)$ & $8(33.3 \%)$ & 0.913 & $12(30.0 \%)$ & $7(29.2 \%)$ & 0.944 \\
\hline $\begin{array}{l}\text { CD4 + T } \\
\text { lymphocytes }\end{array}$ & $21(33.9 \%)$ & $43(32.1 \%)$ & 0.805 & $8(36.4 \%)$ & $9(37.5 \%)$ & 0.936 & $13(32.5 \%)$ & $7(29.2 \%)$ & 0.781 \\
\hline $\begin{array}{l}\text { CD8 + T } \\
\text { lymphocytes }\end{array}$ & $12(19.4 \%)$ & $26(19.4 \%)$ & 0.994 & $3(13.6 \%)$ & $5(20.8 \%)$ & 0.702 & $9(22.5 \%)$ & $6(25.0 \%)$ & 0.819 \\
\hline $\begin{array}{l}\text { Indicators of o } \\
\text { abnormalities; } \\
\text { Respiratory: bc } \\
\text { indicator may }\end{array}$ & $\begin{array}{l}\text { unction i } \\
\text { tive Only } \\
\text { estive an }\end{array}$ & $\begin{array}{l}\text { and immu } \\
\text { rointestina } \\
\text { piratory sy }\end{array}$ & $\begin{array}{l}\text { fllam } \\
\text { nptor } \\
\text { oms. }\end{array}$ & $\begin{array}{l}\text { tion were } \\
\text { cases; Re } \\
\text { cause not }\end{array}$ & $\begin{array}{l}\text { essed in t } \\
\text { tory Only } \\
\text { atients ha }\end{array}$ & $\begin{array}{l}\text { s of th } \\
\text { spirato } \\
\text { the sal }\end{array}$ & $\begin{array}{l}\text { lumber an } \\
\text { symptoms } \\
\text { test, the d }\end{array}$ & $\begin{array}{l}\text { ercentage } \\
\text { ases; Diges } \\
\text { ominators }\end{array}$ & each \\
\hline
\end{tabular}

\section{Mild liver damage and activation of the immunoinflammatory system are the most common manifestations in mild patients on admission}

Mild liver injury was the most common observed in mild patients on admission. In the increased ALT (30.6\%) and AST (15.0\%) level, the mean value was $36.4 \mathrm{U} / \mathrm{L}$ and $31.9 \mathrm{U} / \mathrm{L}$, respectively. Most (17.5\%) of the abnormal ALT and AST are $40 \sim 80 \mathrm{U} / \mathrm{L}$, and those $\geq 80 \mathrm{U} / \mathrm{L}$ are less than $5 \%$. Abnormal LDH was also common accounted for $32.0 \%$. It seems that the proportion of abnormal liver function in the Respiratory only group was slightly higher than that of the other two groups but not statistically significant (Table 2, $p=0.186$ ). The function of bile secretion was also slightly declined with increased levels of TBil and DBil, and increased activities of ALP and GGT. The levels of TP and albumin were slightly decrease which implied hepatic synthetic function was acceptable. Other abnormal creatinine, electrolyte disturbance, troponin, platelets, coagulation indexes and D-dimer were not common. Overall, hepatobiliary system is more vulnerable to damage while renal and cardiac disfunctions were rare in mild patients. 
Table 2

Multiorgan function change characteristics based on the classification of main symptoms in COVID-19 patients on admission.

\begin{tabular}{|c|c|c|c|c|c|}
\hline Items & $\begin{array}{l}\text { Total } \\
(\mathrm{N}=206)\end{array}$ & $\begin{array}{l}\text { Digestive } \\
\text { Only }(\mathrm{N}=48)\end{array}$ & $\begin{array}{l}\text { Respiratory } \\
\text { Only }(\mathrm{N}=89)\end{array}$ & $\begin{array}{l}\text { Digestive + Respiratory } \\
(\mathrm{N}=69)\end{array}$ & $\mathbf{P}$ \\
\hline \multicolumn{6}{|l|}{ Blood Biochemistry } \\
\hline TBil $(\mu \mathrm{mol} / \mathrm{L})$ & $12.9(2.15-35.7)$ & $12.1(6.7-22.9)$ & $13.1(2.15-35.7)$ & $13.2(4.1-26.3)$ & 0.391 \\
\hline$\leq 19.0 \mu \mathrm{mol} / \mathrm{L}$ & 189 (91.7\%) & $44(91.7 \%)$ & $83(93.3 \%)$ & 62 (89.9\%) & 0.743 \\
\hline$>19.0 \mu \mathrm{mol} / \mathrm{L}$ & $17(8.3 \%)$ & $4(8.3 \%)$ & $6(6.7 \%)$ & $7(10.1 \%)$ & \\
\hline DBil $(\mu \mathrm{mol} / \mathrm{L})$ & $4.6(1.7-16.6)$ & $4.2(2.3-9.6)$ & $5.1(2.0-16.6)$ & $4.2(1.7-9.7)$ & 0.008 \\
\hline$\leq 6.8 \mu \mathrm{mol} / \mathrm{L}$ & 185 (89.8\%) & $45(93.8 \%)$ & $76(85.4 \%)$ & 64 (92.8\%) & 0.186 \\
\hline$>6.8 \mu \mathrm{mol} / \mathrm{L}$ & $21(10.2 \%)$ & $3(6.2 \%)$ & $13(14.6 \%)$ & $5(7.2 \%)$ & \\
\hline $\mathrm{TP}(\mathrm{g} / \mathrm{L})$ & $63.5(52.7-81.8)$ & $63.9(54.2-74.5)$ & $62.9(58.8-81.8)$ & $64.0(52.7-76.4)$ & 0.525 \\
\hline$<64 \mathrm{~g} / \mathrm{L}$ & 107 (51.9\%) & $24(50.0 \%)$ & $47(52.8 \%)$ & $36(52.2 \%)$ & 0.951 \\
\hline$\geq 64 \mathrm{~g} / \mathrm{L}$ & $99(48.1 \%)$ & $24(50.0 \%)$ & $42(47.2 \%)$ & $33(47.8 \%)$ & \\
\hline Albumin $(\mathrm{g} / \mathrm{L})$ & $36.5(23.9-68.0)$ & $36.3(27.8-43.8)$ & $36.3(24.6-68.0)$ & $36.9(23.9-47.8)$ & 0.723 \\
\hline$<35 \mathrm{~g} / \mathrm{L}$ & 76 (36.9\%) & $13(27.1 \%)$ & 37 (41.6\%) & $26(37.7 \%)$ & 0.242 \\
\hline$\geq 35 \mathrm{~g} / \mathrm{L}$ & $130(63.1 \%)$ & $35(72.9 \%)$ & $52(58.4 \%)$ & $43(62.3 \%)$ & \\
\hline $\mathrm{ALT}(\mathrm{U} / \mathrm{L})$ & $36.4(7-498)$ & $32.4(7-176)$ & $40.8(7-498)$ & 33.7 (7-152) & 0.229 \\
\hline$\leq 35 \mathrm{U} / \mathrm{L}$ & $143(69.4 \%)$ & $35(72.9 \%)$ & $60(67.4 \%)$ & $48(69.6 \%)$ & 0.800 \\
\hline$>35 \mathrm{U} / \mathrm{L}$ & $63(30.6 \%)$ & $13(27.1 \%)$ & $29(32.6 \%)$ & $21(30.4 \%)$ & \\
\hline AST (U/L) & $31.9(10-346)$ & $29.9(11-81)$ & $35.4(12-346)$ & $28.9(10-135)$ & 0.167 \\
\hline$\leq 40 \mathrm{U} / \mathrm{L}$ & $175(85.0 \%)$ & $41(85.4 \%)$ & $75(84.3 \%)$ & $59(85.5 \%)$ & 0.972 \\
\hline$>40 \mathrm{U} / \mathrm{L}$ & 31 (15.0\%) & $7(14.6 \%)$ & $14(15.7 \%)$ & $10(14.5 \%)$ & \\
\hline $\operatorname{ALP}(\mathrm{U} / \mathrm{L})$ & $72.5(19-250)$ & $76.4(27-214)$ & $75.1(19-250)$ & $66.3(33-141)$ & 0.100 \\
\hline$<150 U / L$ & $203(97.6 \%)$ & $46(95.8 \%)$ & $86(96.6 \%)$ & $69(100.0 \%)$ & 0.123 \\
\hline$>150 U / L$ & $5(2.4 \%)$ & $2(4.2 \%)$ & $3(3.4 \%)$ & $0(0.0 \%)$ & \\
\hline GGT (U/L) & $38.6(7-291)$ & $39.6(8-196)$ & $39.9(7-291)$ & $36.1(11-162)$ & 0.786 \\
\hline$<50 U / L$ & $157(76.2 \%)$ & $39(81.2 \%)$ & $65(73.0 \%)$ & $53(76.8 \%)$ & 0.554 \\
\hline$>50 \mathrm{U} / \mathrm{L}$ & $49(23.8 \%)$ & $9(18.8 \%)$ & $24(27.0 \%)$ & $16(23.2 \%)$ & \\
\hline $\mathrm{LDH}(\mathrm{U} / \mathrm{L})$ & $230.7(15-649)$ & $241.2(88-649)$ & $230.4(15-527)$ & $223.7(114-497)$ & 0.549 \\
\hline$<245 \mathrm{U} / \mathrm{L}$ & $140(68.0 \%)$ & $29(60.4 \%)$ & $63(67.4 \%)$ & $48(69.6 \%)$ & 0.539 \\
\hline$>245 \mathrm{U} / \mathrm{L}$ & $66(32.0 \%)$ & $19(39.6 \%)$ & $29(32.6 \%)$ & $21(30.4 \%)$ & \\
\hline CREA $(44-133 \mu \mathrm{mol} / \mathrm{L})$ & $73.3(39.6-430)$ & $67.8(44.4-121)$ & $79.2(41.6-430)$ & $69.5(39.6-121)$ & 0.128 \\
\hline Glu $(3.9-6.1 \mathrm{mmol} / \mathrm{L})$ & $6.1(1.79-20.06)$ & $5.62(3.95-13.49)$ & $6.4(1.79-15.04)$ & $6.1(3.94-20.06)$ & 0.700 \\
\hline $\mathrm{K}(3.5-5.2 \mathrm{mmol} / \mathrm{L})$ & $4.0(2.8-5.79)$ & $4.1(3.27-5.79)$ & $4.0(2.8-5.79)$ & $4.0(2.8-5.1)$ & 0.276 \\
\hline $\mathrm{Ca}(2.03-2.54 \mathrm{mmol} / \mathrm{L})$ & $2.2(0.2-4.51)$ & $2.2(1.92-3.21)$ & $2.2(1.78-4.51)$ & $2.2(0.2-2.54)$ & 0.642 \\
\hline hsTNI (<26.2 ng/L) & $4.6(0.4-38.0)$ & $4.9(0.5-34.7)$ & $5.1(0.4-38.0)$ & $3.5(0.7-19.2)$ & 0.285 \\
\hline
\end{tabular}




\begin{tabular}{|c|c|c|c|c|c|}
\hline Items & $\begin{array}{l}\text { Total } \\
(\mathrm{N}=206)\end{array}$ & $\begin{array}{l}\text { Digestive } \\
\text { Only }(\mathrm{N}=48)\end{array}$ & $\begin{array}{l}\text { Respiratory } \\
\text { Only }(\mathrm{N}=89)\end{array}$ & $\begin{array}{l}\text { Digestive + Respiratory } \\
(\mathrm{N}=69)\end{array}$ & $\mathbf{P}$ \\
\hline \multicolumn{6}{|c|}{ Blood routine and coagulation function } \\
\hline HGB $(130-175 \mathrm{~g} / \mathrm{L})$ & $122.3(80-159)$ & $119.6(94-151)$ & $122.5(80-161)$ & $123.9(93-159)$ & 0.390 \\
\hline PLT (125-350G/L) & $241.9(24-537)$ & $239.9(91-479)$ & $242.9(24-463)$ & $241.8(102-537)$ & 0.982 \\
\hline АРTT (28-43.5 s) & $37.1(13.7-56.3)$ & $37.3(28.1-55.8)$ & $37.7(26.2-56.3)$ & $36.3(13.7-49.9)$ & 0.329 \\
\hline PT (11-16s) & $13.3(11.4-16.6)$ & $13.4(11.4-15.4)$ & $13.4(11.9-16.6)$ & $13.2(11.7-15.0)$ & 0.237 \\
\hline D-Dimer $(<0.5 \mathrm{ng} / \mathrm{L})$ & $1.0(0.2-20)$ & $1.0(0.2-20)$ & $1.0(0.2-13.01)$ & $0.9(0.2-8.78)$ & 0.383 \\
\hline
\end{tabular}

With regard to inflammatory cytokines (Table 3), 20 (9.7\%) and 69 (33.5\%) patients had neutropenia and lymphopenia, respectively. Procalcitonin was normal in all cases. The Respiratory only group exhibited more enhanced levels of CRP (59.7\% vs.37.2\%) and TNF-a $(7.4 \mathrm{pg} / \mathrm{ml}$ vs. $4.19 \mathrm{pg} / \mathrm{ml}$ ) than those of the Digestive only group (all $\mathrm{P}<0.05)$. Minority of mild patients presented dysregulation of cytokines such as IL-2 (4, 2.1\%), IL-4 (15, 8.0\%), IL-10 (26, 13.8\%) and TNF-a (6, 3.2\%) while almost everyone showed an increase in IL-6 (178, 94.7\%). More importantly, the levels of IL-2 (2.92 pg/ml vs. $2.62 \mathrm{pg} / \mathrm{ml}, \mathrm{P}=0.003)$, IL-4 $(2.57 \mathrm{pg} / \mathrm{ml}$ vs. $2.15 \mathrm{pg} / \mathrm{ml}, \mathrm{P}=0.009)$ and IL-10 $(4.42 \mathrm{pg} / \mathrm{ml}$ vs. $3.75 \mathrm{pg} / \mathrm{ml}, \mathrm{P}=0.08)$ were higher in Digestive only group compared to those of the cases with Respiratory Only. Among groups, no significant differences in immune system parameters were found(Pख0.05). 
Table 3

Inflammation and immune characteristics based on the classification of main symptoms in COVID-19 patients.

\begin{tabular}{|c|c|c|c|c|c|}
\hline Items & Total & GIS & RPS & GRS & $\mathbf{P}$ \\
\hline WBC (G/L) & $\begin{array}{l}5.54(1.73- \\
11.64)\end{array}$ & $\begin{array}{l}5.65(2.66- \\
11.64)\end{array}$ & $\begin{array}{l}5.44(1.73- \\
10.24)\end{array}$ & $\begin{array}{l}5.58(2.42- \\
10.48)\end{array}$ & 0.789 \\
\hline$<9.5 \mathrm{G} / \mathrm{L}$ & $196(95.1 \%)$ & $46(95.8 \%)$ & $84(94.4 \%)$ & $66(95.7 \%)$ & 0.906 \\
\hline$>9.5 \mathrm{G} / \mathrm{L}$ & $10(4.9 \%)$ & $2(4.2 \%)$ & $5(5.6 \%)$ & $3(4.3 \%)$ & \\
\hline Neutrophil (G/L) & $3.58(0.93-8.67)$ & $3.61(1.36-8.1)$ & $3.51(0.93-8.56)$ & $3.65(1.13-8.67)$ & 0.854 \\
\hline$<6.3 \mathrm{G} / \mathrm{L}$ & 191 (92.7\%) & $45(93.7 \%)$ & $82(92.1 \%)$ & $64(92.8 \%)$ & 0.941 \\
\hline$>6.3 \mathrm{G} / \mathrm{L}$ & $15(7.3 \%)$ & $3(6.3 \%)$ & $7(7.9 \%)$ & $5(7.2 \%)$ & \\
\hline Lymphocyte (G/L) & $1.35(0.28-3.4)$ & $1.41(0.42-2.18)$ & $1.28(0.28-3.4)$ & $1.39(0.39-2.76)$ & 0.302 \\
\hline$<1.1 \mathrm{G} / \mathrm{L}$ & $69(33.5 \%)$ & $16(33.3 \%)$ & $34(38.2 \%)$ & $19(27.5 \%)$ & 0.371 \\
\hline$\geq 1.1 \mathrm{G} / \mathrm{L}$ & $137(66.5 \%)$ & $32(66.7 \%)$ & $55(61.8 \%)$ & $50(72.5 \%)$ & \\
\hline Procalcitonin(ug/L) & $0.14(0.03-2.99)$ & $0.13(0.03-2.99)$ & $0.14(0.03-2.99)$ & $0.14(0.03-2.99)$ & 0.139 \\
\hline$\llbracket 0.5 \mathrm{ug} / \mathrm{L}$ & $183(88.8 \%)$ & $42(87.5 \%)$ & $80(89.9 \%)$ & $61(88.4 \%)$ & 0.906 \\
\hline$\geq 0.5 \mathrm{ug} / \mathrm{L}$ & $23(11.2 \%)$ & $6(12.5 \%)$ & $9(10.1 \%)$ & $8(11.6 \%)$ & \\
\hline C-reactive protein(mg/L) & $20.5(0.3-173)$ & $19.3(0.6-173)$ & $26.2(0.3-136)$ & $21.8(0.6-168)$ & 0.569 \\
\hline$\bowtie 8 \mathrm{mg} / \mathrm{L}$ & $90(51.4 \%)$ & $27(62.8 \%)$ & $31(40.3 \%)$ & $32(58.2 \%)$ & 0.029 \\
\hline$\geq 8 \mathrm{mg} / \mathrm{L}$ & $85(48.6 \%)$ & $16(37.2 \%)$ & $46(59.7 \%)$ & $23(41.8 \%)$ & \\
\hline $\mathrm{ESR}(\mathrm{mm} / \mathrm{h})$ & $42.1(2-140)$ & $38.2(7-99)$ & $46.8(2-140)$ & $38.5(2-112)$ & 0.357 \\
\hline$\otimes 15 \mathrm{~mm} / \mathrm{h}$ & $35(19.7 \%)$ & $6(15.0 \%)$ & $14(17.9 \%)$ & $15(25.0 \%)$ & 0.411 \\
\hline$\geq 15 \mathrm{mg} / \mathrm{L}$ & $143(80.3 \%)$ & $34(85.0 \%)$ & $64(82.1 \%)$ & $45(75.0 \%)$ & \\
\hline \multicolumn{6}{|l|}{ Inflammatory cytokines } \\
\hline IL-2(0.1-4.1 pg/ml) & $2.66(2.07-4.93)$ & $2.92(2.07-4.93)$ & $2.62(1.85-4.08)$ & $2.59(1.82-3.98)$ & ¿. 001 \\
\hline $\mathrm{IL}-4(0.1-3.2 \mathrm{pg} / \mathrm{ml})$ & $2.32(0.98-8.23)$ & $2.57(1.04-8.23)$ & $2.15(1.00-4.18)$ & $2.00(0.98-4.80)$ & 0.002 \\
\hline IL-6(0.1-2.9 pg/ml) & $\begin{array}{l}40.09(1.96- \\
437.81)\end{array}$ & $\begin{array}{l}45.53 \text { (1.96- } \\
437.81)\end{array}$ & $\begin{array}{l}43.18(2.27- \\
424.93)\end{array}$ & $\begin{array}{l}31.64 \text { (2.05- } \\
383.95)\end{array}$ & 0.559 \\
\hline$\varangle 2.9 \mathrm{pg} / \mathrm{ml}$ & $10(5.3 \%)$ & $4(8.5 \%)$ & $3(3.7 \%)$ & $3(5.0 \%)$ & 0.610 \\
\hline $2.9-90 \mathrm{pg} / \mathrm{ml}$ & $159(84.6 \%)$ & $37(78.7 \%)$ & $69(85.2 \%)$ & $53(88.3 \%)$ & \\
\hline$\geq 90 \mathrm{pg} / \mathrm{ml}$ & 19(10.1\%) & $6(12.8 \%)$ & $9(11.1 \%)$ & $4(6.7 \%)$ & \\
\hline IL-10(0.1-5.0 pg/ml) & $\begin{array}{l}3.84(1.75- \\
15.98)\end{array}$ & $\begin{array}{l}4.42(2.15- \\
15.98)\end{array}$ & $3.75(1.92-9.34)$ & $3.46(1.75-6.30)$ & 0.021 \\
\hline TNF-a $(0.1-23$ pg/ml) & $5.76(0.90-67.26)$ & $\begin{array}{l}4.19(1.01- \\
21.26)\end{array}$ & $7.40(0.90-67.26)$ & $\begin{array}{l}4.78(1.27- \\
20.73)\end{array}$ & 0.014 \\
\hline \multicolumn{6}{|l|}{ Immune system indexes } \\
\hline $\operatorname{lgG}(7.51-15.6 \mathrm{~g} / \mathrm{L})$ & $11.53(7.3-16.8)$ & $\begin{array}{l}11.39(7.30- \\
16.80)\end{array}$ & $\begin{array}{l}11.67(8.15- \\
16.60)\end{array}$ & $\begin{array}{l}11.56(6.83- \\
19.30)\end{array}$ & 0.912 \\
\hline $\operatorname{lgM}(0.46-3.04 \mathrm{~g} / \mathrm{L})$ & $1.32(23.8 \%)$ & $1.06(0.50-2.31)$ & $\begin{array}{l}1.68(0.39- \\
12.03)\end{array}$ & $1.03(0.46-1.96)$ & 0.200 \\
\hline
\end{tabular}




\begin{tabular}{|c|c|c|c|c|c|}
\hline Items & Total & GIS & RPS & GRS & $\mathbf{P}$ \\
\hline C3(0.79-1.52 g/L) & $0.91(14.6 \%)$ & $0.94(0.64-1.24)$ & $0.90(0.53-1.24)$ & $0.91(0.71-1.10)$ & 0.684 \\
\hline C4(0.16-0.38 g/L) & $0.23(6.4 \%)$ & $0.23(0.14-0.41)$ & $0.24(0.08-0.41)$ & $0.19(0.03-0.29)$ & 0.055 \\
\hline $\begin{array}{l}\mathrm{CD}^{+} \text {Tlymphocytes }(58.17- \\
84.22 \%)\end{array}$ & $\begin{array}{l}73.07(26.12- \\
93.02)\end{array}$ & $\begin{array}{l}73.67(45.44- \\
93.02)\end{array}$ & $\begin{array}{l}72.12(49.40- \\
85.88)\end{array}$ & $\begin{array}{l}73.10(26.12- \\
92.20)\end{array}$ & 0.996 \\
\hline $\begin{array}{l}\text { CD } 4^{+} \mathrm{T} \text { lymphocytes (25.34- } \\
54.37 \%)\end{array}$ & $\begin{array}{l}46.69(10.70- \\
75.73)\end{array}$ & $\begin{array}{l}48.87(24.19- \\
75.73)\end{array}$ & $\begin{array}{l}47.62(35.22- \\
64.48)\end{array}$ & $\begin{array}{l}44.67(10.70- \\
70.46)\end{array}$ & 0.186 \\
\hline $\begin{array}{l}\mathrm{CD}^{+} \mathrm{T} \text { lymphocytes (14.23- } \\
38.95 \%)\end{array}$ & $\begin{array}{l}22.47(5.09- \\
58.88)\end{array}$ & $\begin{array}{l}21.40(7.57- \\
38.74)\end{array}$ & $\begin{array}{l}21.33(7.27- \\
34.99)\end{array}$ & $\begin{array}{l}23.78(5.09- \\
58.88)\end{array}$ & 0.282 \\
\hline
\end{tabular}

\section{Dynamic Longitudinal Changes Of Immune Systems In Mild Patients}

We then analyzed the dynamic changes of white blood cells (WBCs), neutrophils, lymphocytes and different lymphocyte subsets in the peripheral blood of these mild patients. No obvious differences of WBC and neutrophil counts between three groups was found during the course of disease (Fig. 1A and B). The lymphocyte counts in Digestive only groups were higher than Respiratory only group at 4-6 days but without a statistical significance (1.46 vs. $1.10, p=0.153)$ and became close during the following period of disease progression (Fig. 1C). As for lymphocyte subsets, $C D 3+$ and $C D 8+T$ cell counts of patients with digestive symptoms was higher at $7-9$ days $(C D 3+, 77.32$ vs. 61.48 vs. $84.33, p=0.123, C D 8+, 32.87$ vs. 18.37 vs. $40.64, p=0.375)$. At $31-33$ days, a peak value of CD3 + and CD4 + T lymphocytes appeared in Digestive only and Digestive + Respiratory groups compared to Respiratory only group. (CD3+, 82.75 vs. $64.41, p=0.016,81.27$ vs. $64.41, p=0.0002, C D 4+, 59.40$ vs.39.63, $p=0.01,47.06$ vs. $39.63, p=0.141$, Fig. 1D-F). No significant differences in B cell and NK cell counts were observed during the whole course (Fig. 1G and H). The dynamic profile of immunoglobulin and complement levels were also analyzed among the three groups but no significant differences were found (Fig. 2).

\section{Dynamic Longitudinal Changes Of Inflammatory Cytokines In Mild Patients}

We further analyzed the dynamic changes of peripheral IL-2, IL-4, IL-6, IL-10 and TNF-a levels. As is shown in Fig. 3, IL-6 presented a fluctuation during day 19 to 39 , especially in patients with only respiratory symptoms. It was shown a peak at 31-33 days (Fig. 3A). However, compared with the moderate trend of IL-2, IL-4, IL-10 and TNF-a in Respiratory only group, a significant increase of IL-2 and IL-4 in Digestive only group at 34-36 days (IL-2, 4.51 vs. 3.08, $p=0.049, \mathrm{IL}-4,4.63$ vs. 2.67, $p=0.041$, Fig. 3B-E).

\section{Significant linear correlation between inflammatory factors in Digestive only patients}

Finally, we used Pearson correlation analysis to compared the correlation of inflammatory indicators among the three group patients. As shown in Fig. 4, the level of LDH and ESR in the group only/accompanied with respiratory symptoms showed stronger linear correlation(Respiratory Only group: $R^{2}=0.211, R=0.459, P<0.001$; Digestive + Respiratory group: $R^{2}=0.212, R=0.46, P<$ 0.001)than the patients with Digestive Only $\left(R^{2}=0.052, R=0.227 ; P=0.171\right)$. However, compared with Respiratory Only group, Digestive only cases showed stronger correlation and linearity in IL-2 and TNF-a $\left(R^{2}=0.003, R=-0.057 ; P=0.615\right.$ vs. $R^{2}=0.443, R=$ $0.666 ; \mathrm{P}<0.001$, respectively).

\section{Discussion}

In the present study, we compared organ function, inflammation and immune characteristics in sub-groups of patients with mild disease severity divided by whether presenting with digestive symptoms or not. We found that mild liver disorder and activation of the immuno-inflammatory system are the most commonly abnormality in mild patients. Additionally, compared with the 
Respiratory only group, inflammatory markers showed slightly higher and later peak values during the subsequent course of disease in the Digestive group.

Liver disorder is the most commonly abnormality in mild patients in our study. Recently, the lancet also published an article which reported 43 of the 99 cases (43.4\%) of COVID-19 had different degrees of liver function injury[9]. However, those are the characteristics of liver function in critically ill patients. Our study founded that hepatobiliary system is more vulnerable to damage even the patients are relatively mild.

Our results further showed that levels of some inflammatory cytokines such as IL-2, IL-4 and IL-10 in patients with digestive symptoms were slightly higher compared to those with respiratory symptoms and had later peak values during the courses. These might be the reason that patients have a longer delay before viral clearance and more likely to be associated with poorer prognosis compared to patients with respiratory symptoms[18].

Compared with the Respiratory Only group, there was a significant correlation between IL-2 and TNF level in the Digestive Only group. IL-2 has an important role in inducing and enhancing cytotoxic activity. Early studies presented a model of T cell differentiation characterized by a switch from "antigen mode", where antigen driven IL-2 expression is abundant, to "inflammation mode", where responses to antigen signals are attenuated, IL-2 gene transcription is deficient, and responses to cytokine signals may predominate[19]. And TNF regulates the switch from "antigen mode" to "inflammation mode" during terminal T cell differentiation[20]. Therefore, these seem to suggest that the appearance of different symptoms may be the comprehensive manifestation of different action mechanisms and different action results. It provides a new way for us to further study the pathogenesis and treatment of the disease. The mechanism of gastrointestinal symptoms and its significance still need to be further studied.

Our study has some notable limitations. First, it would be better to include as many patients as possible in Wuhan, in other cities in China, and even in other countries to get a more comprehensive understanding of 2019nCoV with digestive system. However, the data in this study permit an early assessment of the inflammatory immune characteristics of gastrointestinal findings with COVID19. Second, no data were included on critically ill patients. However, the aim to study immune inflammatory characteristics in mild cases might be our advantage. Third, there were not many obvious differences in the longitudinal analysis were found in immunoinflammatory characteristics. Further study focused on these aspects are needed.

In conclusion, patients only/with gastrointestinal symptoms are a special group of COVID-19. Patients in this group were more likely to have slightly higher and later peak values of inflammatory cytokines during the subsequent course of disease. The prevention and clinical management of this type should be taken into consideration.

\section{Conclusions}

In conclusion, mild patients with digestive symptoms are a special subtype of COVID-19. This kind of patients were more likely to have slightly higher and later peak values of inflammatory cytokines during the subsequent course of disease. The prevention and clinical management of this type should be taken into consideration.

\section{List Of Abbreviations}

COVID-19: Coronavirus disease 2019

ACE2: angiotensin converting enzyme 2

hCoVs: human coronaviruses

SARS-CoV: severe acute respiratory syndrome coronavirus

MERS-CoV: middle east respiratory syndrome coronavirus

\section{Declarations}

Page 10/16 
Conflict of Interest Statement

All authors state that they have no conflict of interest.

Financial support:

This study was supported by grants of a novel coronavirus pneumonia emergency science and technology projects from the Science and Technology Department of Hubei Province, Wuhan, China (2020FCA014). And part was supported by the National Natural Science Foundation of China (No. 81720108006, 81800467, 81974062).

\section{Author contribution}

A. Duan and S. Zhang collected medical records data, analyzed the data, drafted the manuscript and contributed equally to this paper. Wang J helped for data statistics. W. Qian supported data entry and sorting; Hou X contributed revisions of the manuscript for important intellectual content. C. Han designed, supervised the study and revised the manuscript as the corresponding author.

\section{Acknowledgements}

We would like to express our heartfelt thanks to those on the front lines of this struggle: medical staff, community workers, cleaners, drivers, construction workers, and all the volunteers involved. We are also very grateful to those who sacrifice their freedom and isolated themselves to break the chain of transmission.

\section{Declarations}

This study was approved by the Medical Ethical Review Committee, Union Hospital of Tongji Medical College, Huazhong University of Science and Technology, China ([2020] No.0033). Written informed consent was waived due to the rapid emergence of this infectious disease and their information had been anonymized and de-identified.

Availability of data and materials

The datasets used and/or analysed during the current study are available from the corresponding author on reasonable request.

\section{References}

1. Li Q, Guan X, Wu P, Wang X, Zhou L, Tong Y, Ren R, Leung KSM, Lau EHY, Wong JY et al: Early Transmission Dynamics in Wuhan, China, of Novel Coronavirus-Infected Pneumonia. The New England journal of medicine 2020, 382(13):1199-1207.

2. Chen L, Liu W, Zhang Q, Xu K, Ye G, Wu W, Sun Z, Liu F, Wu K, Zhong B et al: RNA based mNGS approach identifies a novel human coronavirus from two individual pneumonia cases in 2019 Wuhan outbreak. Emerging microbes \& infections 2020, 9(1):313-319.

3. Jin YH, Cai L, Cheng ZS, Cheng H, Deng T, Fan YP, Fang C, Huang D, Huang LQ, Huang Q et al: A rapid advice guideline for the diagnosis and treatment of 2019 novel coronavirus (2019-nCoV) infected pneumonia (standard version). Military Medical Research 2020, 7(1):4.

4. Wong SH, Lui RN, Sung JJ: Covid-19 and the Digestive System. Journal of gastroenterology and hepatology 2020.

5. Zhou Z, Zhao N, Shu Y, Han S, Chen B, Shu X: Effect of gastrointestinal symptoms on patients infected with COVID-19. Gastroenterology 2020.

6. ACE2 angiotensin I converting enzyme 2 [ Homo sapiens (human)] [https://www.ncbi.nlm.nih.gov/gene/59272]

7. Xiao F, Tang M, Zheng X, Liu Y, Li X, Shan H: Evidence for gastrointestinal infection of SARS-CoV-2. Gastroenterology 2020.

8. Chen L, Lou J, Bai Y, Wang M: COVID-19 Disease With Positive Fecal and Negative Pharyngeal and Sputum Viral Tests. The American journal of gastroenterology 2020.

9. Chen N, Zhou M, Dong X, Qu J, Gong F, Han Y, Qiu Y, Wang J, Liu Y, Wei Y et al: Epidemiological and clinical characteristics of 99 cases of 2019 novel coronavirus pneumonia in Wuhan, China: a descriptive study. Lancet (London, England) 2020, 395(10223):507-513. 
10. [The epidemiological characteristics of an outbreak of 2019 novel coronavirus diseases (COVID-19) in China]. Zhonghua liu xing bing xue za zhi = Zhonghua liuxingbingxue zazhi 2020, 41(2):145-151.

11. Chaoqun Han CD, Shengyan Zhang, Brennan Spiegel, Huiying Shi,Weijun Wang, Lei Zhang, Rong Lin, Jun Liu, Zhen Ding, Xiaohua Hou: Digestive Symptoms in COVID-19 Patients with Mild Disease Severity: Clinical Presentation, Stool Viral RNA Testing, and Outcomes. The American journal of gastroenterology 2020.

12. Lei Pan M, PhD, Mi Mu, MD, Pengcheng Yang, MD, Yu Sun, MD, Runsheng, Wang, MS, Junhong Yan, MD, Pibao Li, MD, Baoguang Hu, MD, PhD, Jing Wang, MS,Chao Hu, MS, Yuan Jin, MD, Xun Niu, MD, Rongyu Ping, MD, Yingzhen Du, MD,Tianzhi $\mathrm{Li}, \mathrm{MD}$, Guogang Xu, MD, PhD, Qinyong Hu, MD, Lei Tu, MD, PhD: Clinical characteristics of COVID-19 patients with digestive symptoms in Hubei, China: a descriptive, cross-sectional, multicenter study. The American journal of gastroenterology March 2020.

13. Channappanavar R, Perlman S: Pathogenic human coronavirus infections: causes and consequences of cytokine storm and immunopathology. Seminars in immunopathology 2017, 39(5):529-539.

14. Chen G, Wu D, Guo W, Cao Y, Huang D, Wang H, Wang T, Zhang X, Chen H, Yu H et al: Clinical and immunologic features in severe and moderate Coronavirus Disease 2019. The Journal of clinical investigation 2020.

15. Xiong Y, Liu Y, Cao L, Wang D, Guo M, Jiang A, Guo D, Hu W, Yang J, Tang Z et al: Transcriptomic characteristics of bronchoalveolar lavage fluid and peripheral blood mononuclear cells in COVID-19 patients. Emerging microbes \& infections 2020, 9(1):761-770.

16. Huang C, Wang Y, Li X, Ren L, Zhao J, Hu Y, Zhang L, Fan G, Xu J, Gu X et al: Clinical features of patients infected with 2019 novel coronavirus in Wuhan, China. Lancet (London, England) 2020, 395(10223):497-506.

17. Patel A, Jernigan DB: Initial Public Health Response and Interim Clinical Guidance for the $\mathbf{2 0 1 9}$ Novel Coronavirus Outbreak United States, December 31, 2019-February 4, 2020. MMWR Morbidity and mortality weekly report 2020, 69(5):140-146.

18. Clinical characteristics of COVID-19 patients with digestive symptoms in Hubei, China: a descriptive, cross-sectional, multicenter study. The American Journal of Gastroenterology, March 2020 (pre-print online) [https://journals.Iww.com/ajg/Documents/COVID_Digestive_Symptoms_AJG_Preproof.pdf]

19. Rosenberg SA: IL-2: the first effective immunotherapy for human cancer. Journal of immunology (Baltimore, Md : 1950) 2014, 192(12):5451-5458.

20. Clark J, Vagenas P, Panesar M, Cope AP: What does tumour necrosis factor excess do to the immune system long term? Annals of the rheumatic diseases 2005, 64 Suppl 4:iv70-76.

\section{Figures}




\section{Figure1}

A

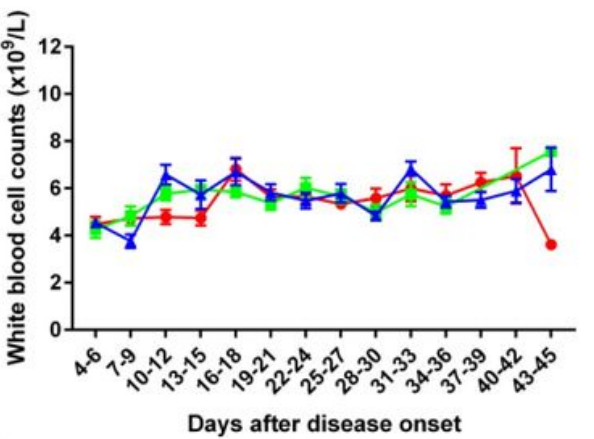

D

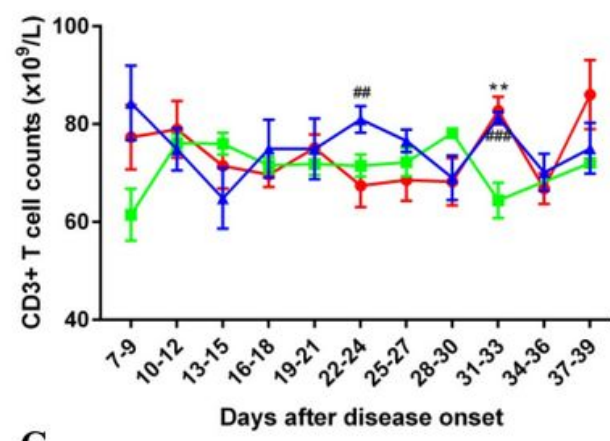

G

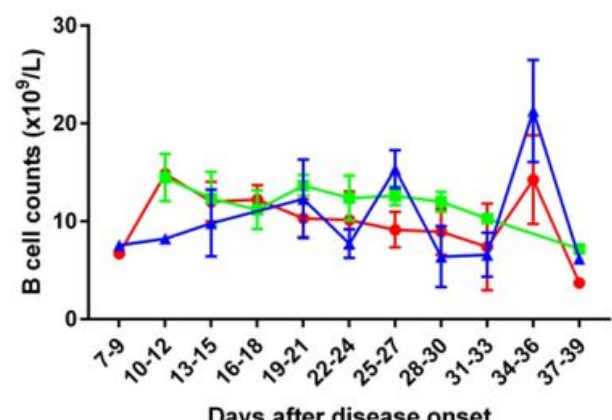

B

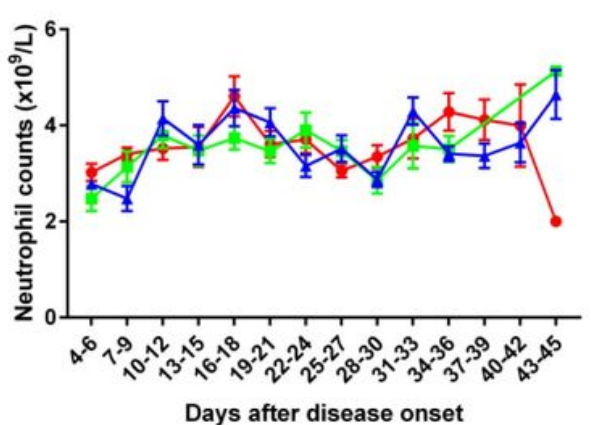

$\mathbf{E}$

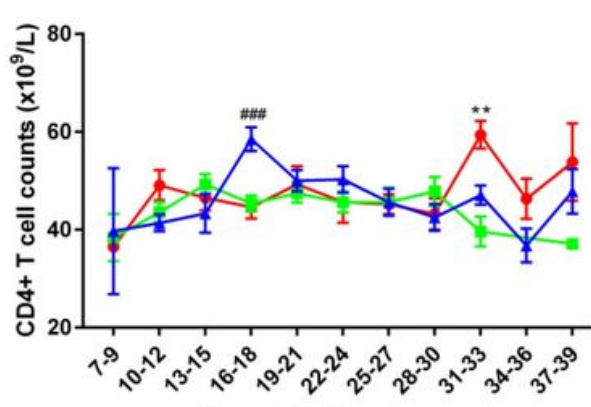

H
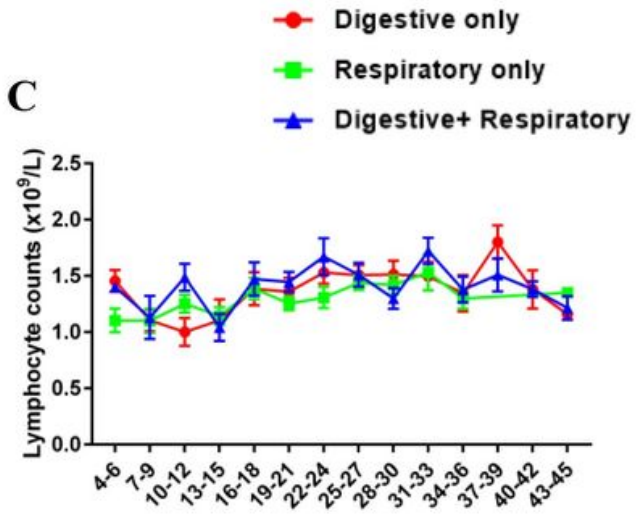

Days after disease onset

$\mathbf{F}$

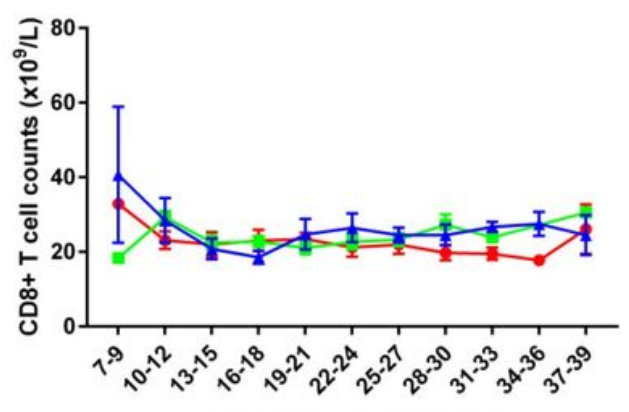

Days after disease onset

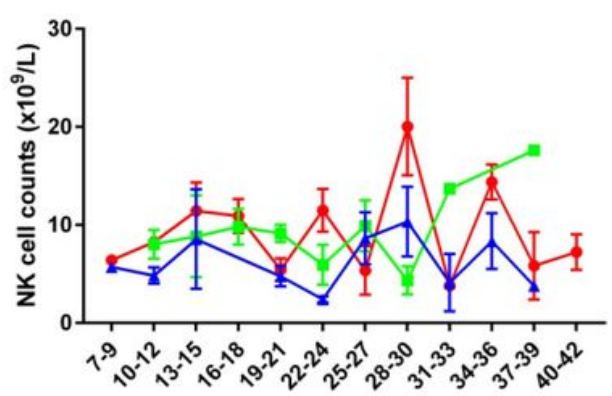

Days after disease onset

Figure 1

Dynamic profile of peripheral immune cells subsets in COVID-19 patients with different symptoms. Timeline charts illustrated the peripheral WBCs (A), Neutrophils (B), Lymphocytes (C), CD3+ T cell (D), CD4+ T cell (E), CD8+ T cell (F), B cell (G) and NK cell (H). Error bars, mean $\pm S E M ; * * P<0.01$, Digestive only vs. Respiratory only. \#\# $P<0.01$, \#\#\# $P<0.001$, Digestive+Respiratory vs. Respiratory only. 
Figure2

A

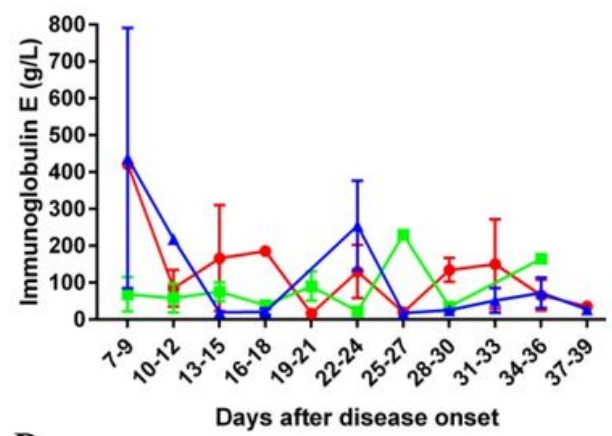

D

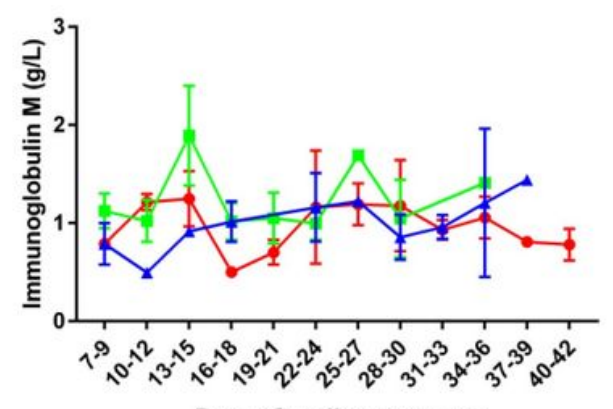

Days after disease onset
B

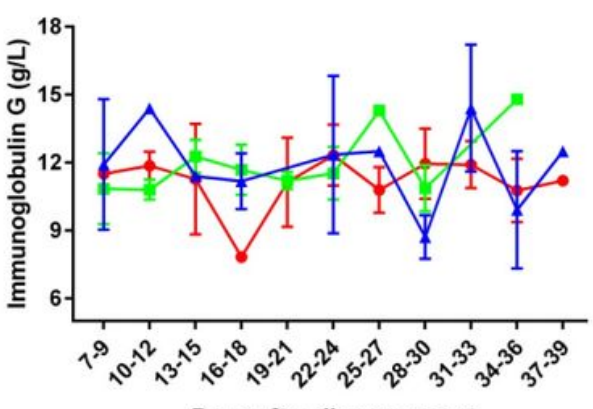

E

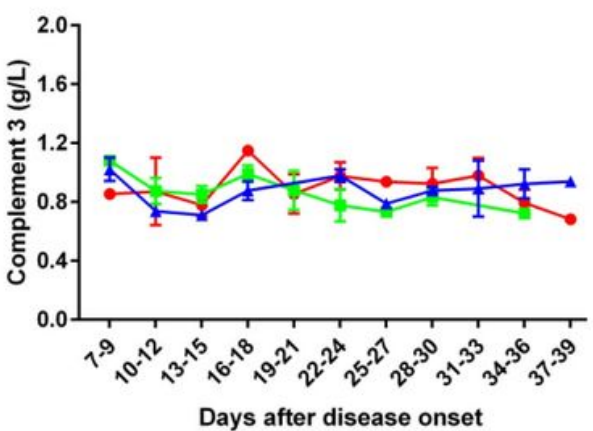

Digestive only

Respiratory only

C

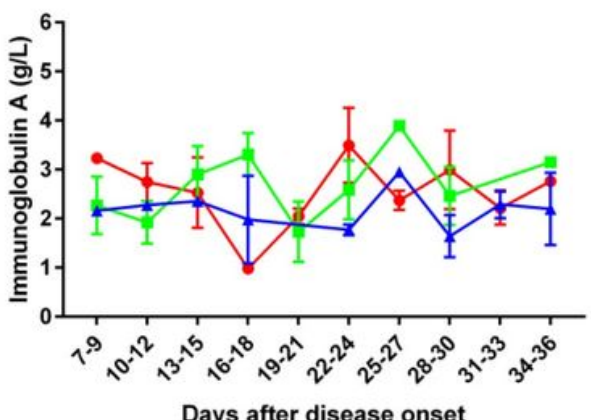

F

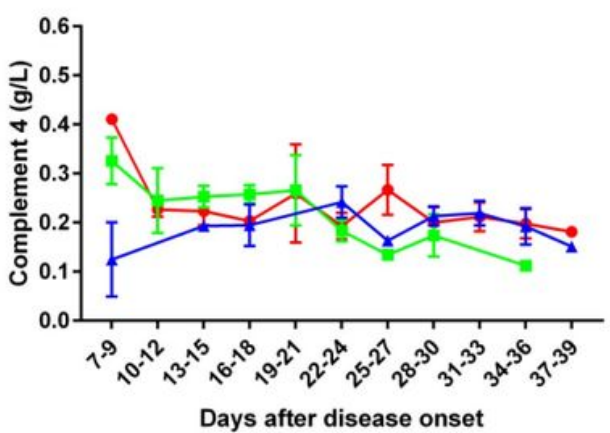

Figure 2

Dynamic profile of immunoglobulin and complement levels in COVID-19 patients with different symptoms. Timeline charts illustrate the levels of $\lg E(A), \lg G(B), \lg A(C), \lg M(D), C 3(E)$ and C4 (F). Error bars, mean $\pm S E M ; * \star P<0.01$, Digestive only vs. Respiratory only. \#\# $\mathrm{P}<0.01$, \#\#\# $\mathrm{P}<0.001$. 
Figure3

A

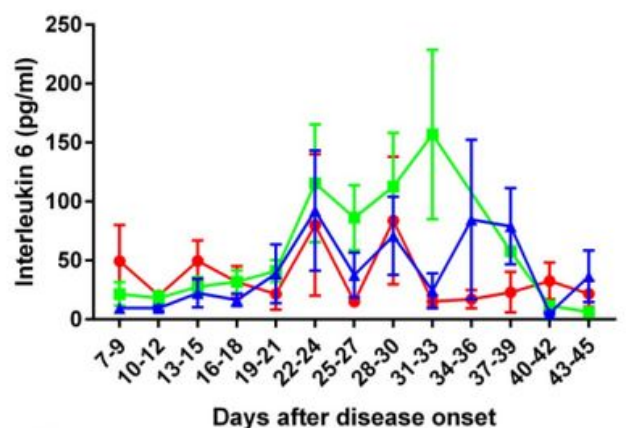

D

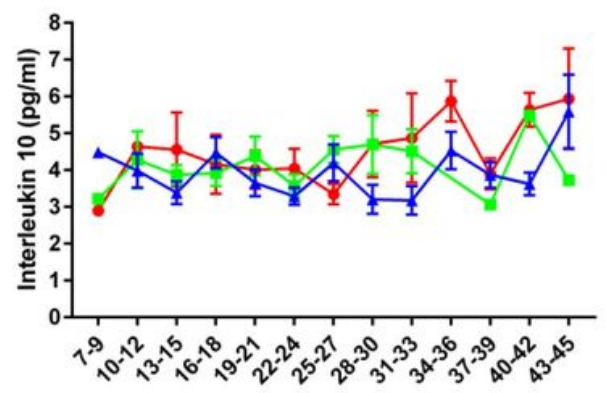

Days after disease onset
B

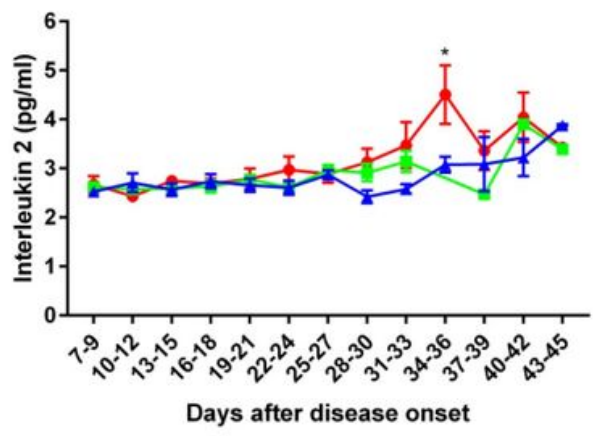

$\mathbf{E}$

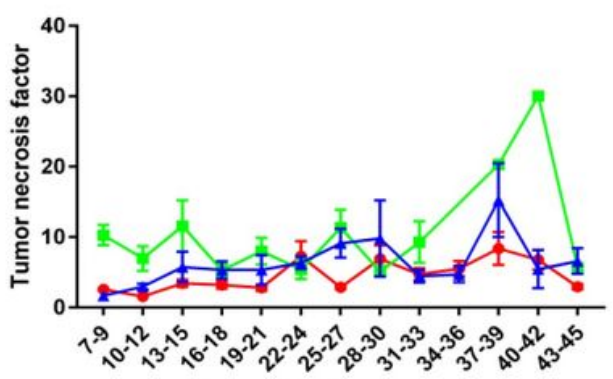

Days after disease onset
C

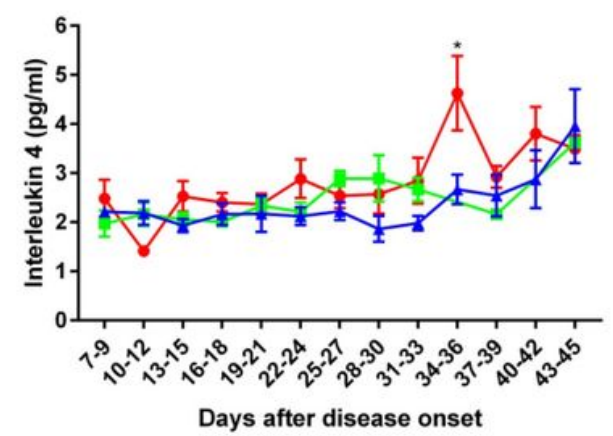

\section{Figure 3}

Dynamic profile of peripheral cytokine levels in COVID-19 patients with different symptoms. Timeline charts illustrate the peripheral IL-6 (A), IL-2 (B), IL-4 (C), IL-10 (D) and TNF-a (E). Error bars, mean \pm SEM.; *P<0.05, Digestive only vs. Digestive+Respiratory. 


\section{Figure4}

A

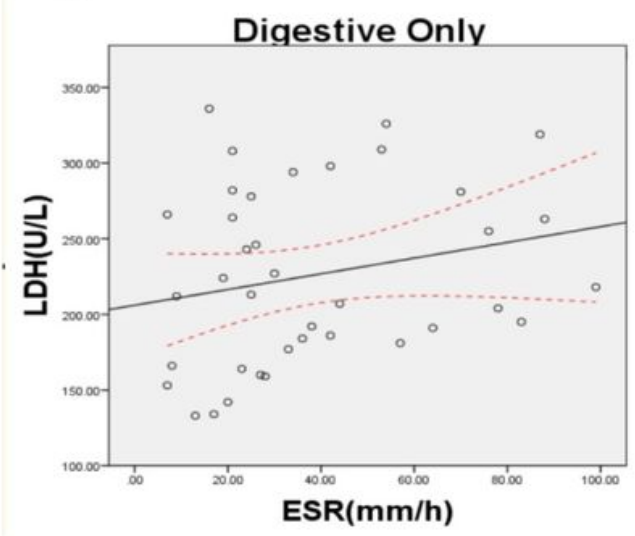

D

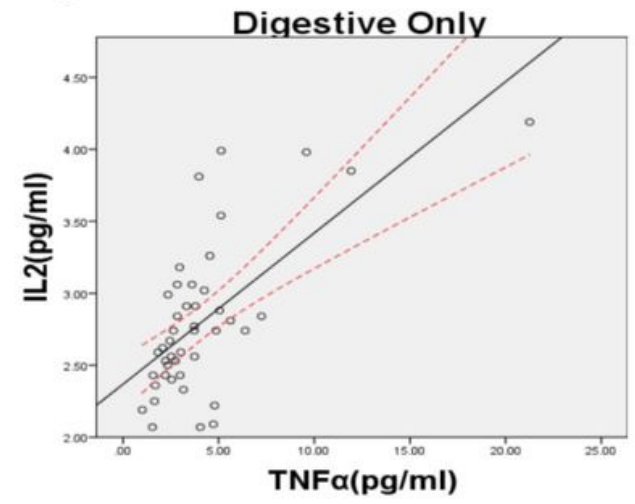

B

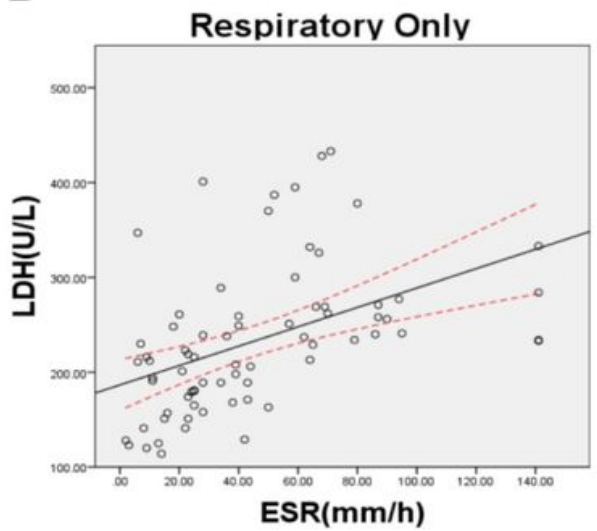

E

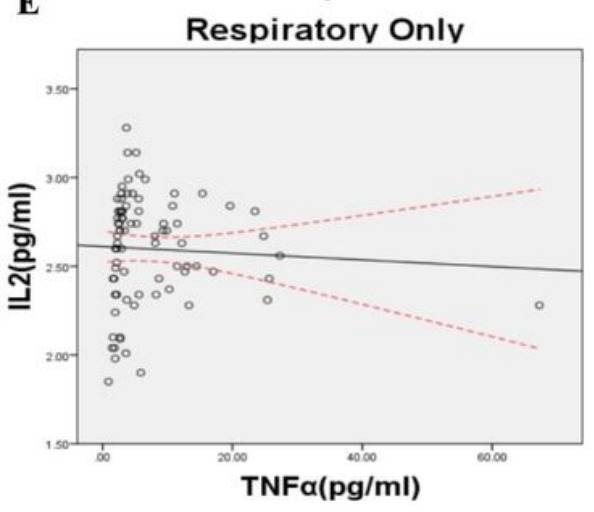

C

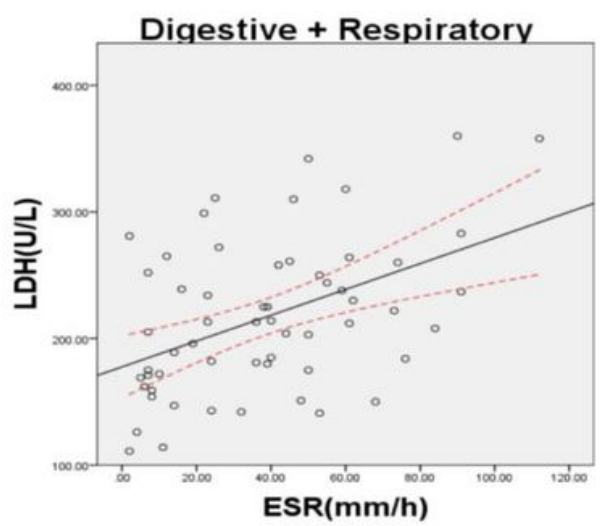

F

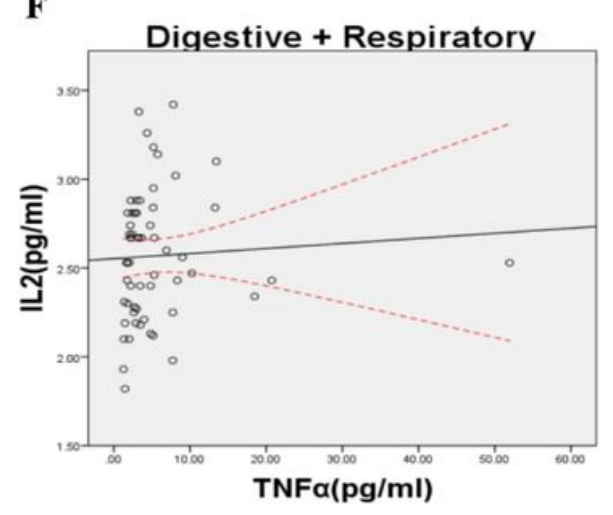

\section{Figure 4}

Correlation analysis of IL-2 levels and TNF-a levels(A,B,C),LDH levels and ESR levels(D,E,F) in COVID-19 patients with different symptoms. The black line is a linear fitting line and the red dotted line represents the confidence interval of the average. 